Proyecciones Journal of Mathematics

Vol. 33, No 1, pp. 91-100, March 2014.

Universidad Católica del Norte

Antofagasta - Chile

\title{
Titchmarsh's Theorem for the Dunkl transform in the space $L^{2}\left(\mathbf{R}^{d}, w_{k}(x) d x\right)$
}

\author{
Radouan Daher \\ and \\ Mohamed El Hamma \\ University of Hassan II, Morocco \\ Received : February 2013. Accepted : August 2013
}

\begin{abstract}
Using a generalized spherical mean operator, we obtain a generalization of Titchmarsh's theorem for the Dunkl transform for functions satisfying the $(\psi, \alpha, \beta)$-Dunkl Lipschitz condition in $L^{2}\left(\mathbf{R}^{d}, w_{k}(x) d x\right)$.
\end{abstract}

Keywords : Dunkl operator, Dunkl transform, generalized spherical mean operator.

Mathematics Subject Classification : 47B48; 33C52. 


\section{Intoduction and preliminaries}

In [10], E. C. Titchmarsh characterized the set of functions in $L^{2}(\mathbf{R})$ satisfying the Cauchy Lipschitz condition for the Fourier transform, namely we have

Theorem 1.1. Let $\alpha \in(0,1)$ and assume that $f \in L^{2}(\mathbf{R})$. Then the following are equivalents

1. $\|f(x+h)-f(x)\|_{L^{2}(\mathbf{R})}=O\left(h^{\alpha}\right)$ as $h \longrightarrow 0$,

2. $\int_{|\lambda| \geq r}|F(\lambda)|^{2} d \lambda=O\left(r^{-2 \alpha}\right)$ as $r \longrightarrow+\infty$,

where $F$ stands for the Fourier transform of $f$.

The main aim of this paper is to establish a generalization of Theorem 1.1 in the Dunkl transform setting by means of the generalized spherical mean operator.

In this paper we consider the Dunkl operators $T_{j}, j=1,2, \ldots, d$, which are the differential-difference operators introduced by C.F. Dunkl in [3]. These operators are very important in pure mathematics and in physics.

In the first we collect some notations and results on Dunkl operators and the Dunkl kernel (see [3], [4], [6]).

We consider $\mathbf{R}^{d}$ with the Euclidean scalar product $\langle.,$.$\rangle and |x|=$ $\sqrt{\langle x, x\rangle}$. For $\alpha \in \mathbf{R}^{d} \backslash\{0\}$, let $\sigma_{\alpha}$ be the reflection in the hyperplane $H_{\alpha} \subset \mathbf{R}^{d}$ orthogonal to $\alpha$.

i.e.,

$$
\sigma_{\alpha}(x)=x-2 \frac{\langle\alpha, x\rangle}{|x|^{2}} \alpha .
$$

A finite set $R \subset \mathbf{R}^{d} \backslash\{0\}$ is called a root system, if $R \cap \mathbf{R} \cdot \alpha=\{\alpha,-\alpha\}$ and $\sigma_{\alpha} R=R$ for all $\alpha \in R$. For a given root system $R$ the reflection $\sigma_{\alpha}, \alpha \in R$, generate a finite group $W \subset O(d)$, the reflection group associated with $R$. We fix $\beta \in \mathbf{R}^{d} \backslash \cup_{\alpha \in R} H_{\alpha}$ and define a positive root system $R_{+}=\{\alpha \in R /\langle\alpha, \beta\rangle>0\}$.

A function $k: R \longrightarrow \mathbf{C}$ on a root system $R$ is called a multiplicity function, if it is invariant under the action of the associated reflection group $W$. 
If one regards $k$ as a function on the corresponding reflections, this means that $k$ is constant on the conjugacy classes of reflections in $W$.

We consider the weight function

$$
w_{k}(x)=\prod_{\alpha \in R_{+}}|\langle\alpha, x\rangle|^{2 k(\alpha)},
$$

where $w_{k}$ is $W$-invariant and homogeneous of degree $2 \gamma$ where

$$
\gamma=\sum_{\alpha \in R_{+}} k(\alpha)
$$

We let $\eta$ be the normalized surface measure on the unit sphere $\mathbf{S}^{d-1}$ in $\mathbf{R}^{d}$ and set

$$
d \eta_{k}(y)=w_{k}(y) d \eta(y) .
$$

Then $\eta_{k}$ is a $W$-invariant measure on $\mathbf{S}^{d-1}$, we let $d_{k}=\eta_{k}\left(\mathbf{S}^{d-1}\right)$.

Introduced by C.F. Dunkl in [3] the Dunkl operators $T_{j}, 1 \leq j \leq d$, on $\mathbf{R}^{d}$ associated with the reflection group $W$ and the multiplicity function $k$ are the first-order differential-difference operators given by

$$
T_{j} f(x)=\frac{\partial f}{\partial x_{j}}(x)+\sum_{\alpha \in R_{+}} k(\alpha) \alpha_{j} \frac{f(x)-f\left(\sigma_{\alpha}(x)\right)}{\langle\alpha, x\rangle}, \quad f \in C^{1}\left(\mathbf{R}^{d}\right),
$$

where $\alpha_{j}=\left\langle\alpha, e_{j}\right\rangle ;\left(e_{1}, \ldots, e_{d}\right)$ being the canonical basis of $\mathbf{R}^{d}$ and $C^{1}\left(\mathbf{R}^{d}\right)$ is the space of functions of class $C^{1}$ on $\mathbf{R}^{d}$.

The Dunkl kernel $E_{k}$ on $\mathbf{R}^{d} \times \mathbf{R}^{d}$ has been introduced by C.F. Dunkl in [5]. For $y \in \mathbf{R}^{d}$ the function $x \longmapsto E_{k}(x, y)$ can be viewed as the solution on $\mathbf{R}^{d}$ of the following initial problem

$$
\begin{cases}T_{j} u(x, y)=y_{j} u(x, y) & \text { for } 1 \leq j \leq d \\ u(0, y)=1 & \text { for all } y \in \mathbf{R}^{d}\end{cases}
$$

This kernel has unique holomorphic extension to $\mathbf{C}^{d} \times \mathbf{C}^{d}$.

M. Rösler has proved in [8] the following integral representation for the Dunkl kernel 


$$
E_{k}(x, z)=\int_{\mathbf{R}^{d}} e^{\langle y, z\rangle} d \mu_{x}(y), x \in \mathbf{R}^{d}, z \in \mathbf{C}^{d}
$$

where $\mu_{x}$ is a probability measure on $\mathbf{R}^{d}$ with support in the closed ball $B(0,|x|)$ of center 0 and raduis $|x|$.

Proposition 1.2. [6]: Let $z, w \in \mathbf{C}^{d}$ and $\lambda \in \mathbf{C}$. Then

1. $E_{k}(z, 0)=1$,

2. $E_{k}(z, w)=E_{k}(w, z)$,

3. $E_{k}(\lambda z, w)=E_{k}(z, \lambda w)$,

4. For all $\nu=\left(\nu_{1}, \ldots . ., \nu_{d}\right) \in \mathbf{N}^{\mathbf{d}}, x \in \mathbf{R}^{d}, z \in \mathbf{C}^{d}$, we have

$$
\left|D_{z}^{\nu} E_{k}(x, z)\right| \leq|x|^{|\nu|} \exp (|x||R e z|)
$$

where

$$
D_{z}^{\nu}=\frac{\partial^{|\nu|}}{\partial z_{1}^{\nu_{1}} \ldots . . \partial z_{d}^{\nu_{d}}} ;|\nu|=\nu_{1}+\ldots+\nu_{d}
$$

In particulier

$$
\left|D_{z}^{\nu} E_{k}(i x, z)\right| \leq|x|^{|\nu|}
$$

for all $x, z \in \mathbf{R}^{d}$.

The Dunkl transform is defined for $f \in L_{k}^{1}\left(\mathbf{R}^{d}\right)=L^{1}\left(\mathbf{R}^{d}, w_{k}(x) d x\right)$ by

$$
\widehat{f}(\xi)=c_{k}^{-1} \int_{\mathbf{R}^{d}} f(x) E_{k}(-i \xi, x) w_{k}(x) d x
$$

where the constant $c_{k}$ is given by

$$
c_{k}=\int_{\mathbf{R}^{d}} e^{-\frac{|z|^{2}}{2}} w_{k}(z) d z
$$

According to $[4,6,9]$ we have the following results: 
1. When both $f$ and $\widehat{f}$ are in $L_{k}^{1}\left(\mathbf{R}^{d}\right)$, we have the inversion formula

$$
f(x)=\int_{\mathbf{R}^{d}} \widehat{f}(\xi) E_{k}(i x, \xi) w_{k}(\xi) d \xi, \quad x \in \mathbf{R}^{d},
$$

2. (Plancherel's theorem) The Dunkl transform on $S\left(\mathbf{R}^{d}\right)$, the space of Schwartz functions, extends uniquely to an isometric isomorphism on $L_{k}^{2}\left(\mathbf{R}^{d}\right)$.

K. Trimèche has introduced [11] the Dunkl translation operators $\tau_{x}, x \in$ $\mathbf{R}^{d}$. For $f \in L_{k}^{2}\left(\mathbf{R}^{d}\right)$ and we have

$$
\left.\widehat{\left(\tau_{x}(f)\right.}\right)(\xi)=E_{k}(i x, \xi) \widehat{f}(\xi)
$$

and

$$
\tau_{x}(f)(y)=c_{k}^{-1} \int_{\mathbf{R}^{d}} \widehat{f}(\xi) E_{k}(i x, \xi) E_{k}(i y, \xi) w_{k}(\xi) d \xi .
$$

The generalized spherical mean operator for $f \in L_{k}^{2}\left(\mathbf{R}^{d}\right)$ is defined by

$$
M_{h} f(x)=\frac{1}{d_{k}} \int_{\mathbf{S}^{d-1}} \tau_{x}(h y) d \eta_{k}(y), x \in \mathbf{R}^{d}, h>0 .
$$

From [7], we have $M_{h} f \in L_{k}^{2}\left(\mathbf{R}^{d}\right)$ whenever $f \in L_{k}^{2}\left(\mathbf{R}^{d}\right)$ and

$$
\left\|M_{h} f\right\|_{L_{k}^{2}} \leq\|f\|_{L_{k}^{2}} .
$$
by

For $p \geq-\frac{1}{2}$, we introduce the normalized Bessel functuion $j_{p}$ defined

$$
j_{p}(z)=\Gamma(p+1) \sum_{n=0}^{\infty} \frac{(-1)^{n}(z / 2)^{2 n}}{n ! \Gamma(n+p+1)}, z \in \mathbf{C},
$$

where $\Gamma$ is the gamma-function.

Lemma 1.3. [1] The following inequalities are fulfilled

1. $\left|j_{p}(x)\right| \leq 1$,

2. $1-j_{p}(x)=O\left(x^{2}\right) ; 0 \leq x \leq 1$. 
Lemma 1.4. The following inequality is true

$$
\left|1-j_{p}(x)\right| \geq c,
$$

with $|x| \geq 1$, where $c>0$ is a certain constant.

(Analog of lemma 2.9 in [2])

Proposition 1.5. Let $f \in L_{k}^{2}\left(\mathbf{R}^{d}\right)$. Then

$$
\left(\widehat{M_{h}} f\right)(\xi)=j_{\gamma+\frac{d}{2}-1}(h|\xi|) \widehat{f}(\xi) .
$$

(See [7])

For any function $f(x) \in L_{k}^{2}\left(\mathbf{R}^{d}\right)$ we define differences of the order $m$ $(m \in\{1,2, \ldots\})$ with a step $h>0$.

$$
\Delta_{h}^{m} f(x)=\left(M_{h}-I\right)^{m} f(x),
$$

here $I$ is the unit operator.

Lemma 1.6. Let $f \in L_{k}^{2}\left(\mathbf{R}^{d}\right)$. Then

$$
\left\|\Delta_{h}^{m} f(x)\right\|_{L_{k}^{2}}^{2}=\int_{\mathbf{R}^{d}}\left|1-j_{\gamma+\frac{d}{2}-1}(h|\xi|)\right|^{2 m}|\widehat{f}(\xi)|^{2} w_{k}(\xi) d \xi .
$$

From formula (1.1) and proposition 1.5, we have

$$
\left(\widehat{\Delta_{h}^{m}} f\right)(\xi)=\left(j_{\gamma+\frac{d}{2}-1}(h|\xi|)-1\right)^{m} \widehat{f}(\xi) .
$$

By Parseval's identity, we obtain

$$
\left\|\Delta_{h}^{m} f(x)\right\|_{L_{k}^{2}}^{2}=\int_{\mathbf{R}^{d}}\left|1-j_{\gamma+\frac{d}{2}-1}(h|\xi|)\right|^{2 m}|\widehat{f}(\xi)|^{2} w_{k}(\xi) d \xi .
$$

The lemma is proved

\section{Main Results}

In this section we give the main result of this paper. We need first to define $(\psi, \alpha, \beta)$-Dunkl Lipschitz class. 
Definition 2.1. Let $\alpha>0$ and $\beta>0$. A function $f \in L_{k}^{2}\left(\mathbf{R}^{d}\right)$ is said to be in the $(\psi, \alpha, \beta)$-Dunkl Lipschitz class, denoted by $\operatorname{Lip}(\psi, \alpha, \beta)$; if:

$$
\left\|\Delta_{h}^{m} f(x)\right\|_{L_{k}^{2}}=O\left(h^{\alpha} \psi\left(h^{\beta}\right)\right) \text { as } h \longrightarrow 0 ; m \in\{1,2, \ldots\},
$$

where $\psi(t)$ is a continuous increasing function on $[0, \infty), \psi(0)=0$ and $\psi(t s)=\psi(t) \psi(s)$ for all $t, s \in[0, \infty)$ and this function verify

$$
\int_{0}^{1 / h} r^{2 m-2 \alpha-1} \psi\left(r^{-2 \beta}\right) d r=O\left(h^{2 \alpha-2 m} \psi\left(h^{2 \beta}\right)\right) \text { as } h \longrightarrow 0 .
$$

Theorem 2.2. Let $f \in L_{k}^{2}\left(\mathbf{R}^{d}\right)$. Then the following are equivalents

1. $f \in \operatorname{Lip}(\psi, \alpha, \beta)$,

2. $\int_{|\xi| \geq s}|\widehat{f}(\xi)|^{2} w_{k}(\xi) d \xi=O\left(s^{-2 \alpha} \psi\left(s^{-2 \beta}\right)\right)$ as $s \longrightarrow+\infty$.

$1) \Longrightarrow 2)$ Assume that $f \in \operatorname{Lip}(\psi, \alpha, \beta)$. Then we have

$$
\left\|\Delta_{h}^{m} f(x)\right\|_{L_{k}^{2}}=O\left(h^{\alpha} \psi\left(h^{\beta}\right)\right) \text { as } h \longrightarrow 0,
$$

From lemma 1.6, we have

$$
\left\|\Delta_{h}^{m} f(x)\right\|_{L_{k}^{2}}^{2}=\int_{\mathbf{R}^{d}}\left|1-j_{\gamma+\frac{d}{2}-1}(h|\xi|)\right|^{2 m}|\widehat{f}(\xi)|^{2} w_{k}(\xi) d \xi .
$$

If $|\xi| \in\left[\frac{1}{h}, \frac{2}{h}\right]$ then $h|\xi| \geq 1$ and lemma 1.4 implies that

$$
1 \leq \frac{1}{c^{2 m}}\left|1-j_{\gamma+\frac{d}{2}-1}(h|\xi|)\right|^{2 m} .
$$

Then

$$
\begin{aligned}
\int_{\frac{1}{h} \leq|\xi| \leq \frac{2}{h}}|\widehat{f}(\xi)|^{2} w_{k}(\xi) d \xi & \leq \frac{1}{c^{2 m}} \int_{\frac{1}{h} \leq|\xi| \leq \frac{2}{h}}\left|1-j_{\gamma+\frac{d}{2}-1}(h|\xi|)\right|^{2 m}|\widehat{f}(\xi)|^{2} w_{k}(\xi) d \xi \\
& \leq \frac{1}{c^{2 m}} \int_{\mathbf{R}^{d}}\left|1-j_{\gamma+\frac{d}{2}-1}(h|\xi|)\right|^{2 m}|\widehat{f}(\xi)|^{2} w_{k}(\xi) d \xi \\
& \leq C h^{2 \alpha}\left(\psi\left(h^{\beta}\right)\right)^{2}=C h^{2 \alpha} \psi\left(h^{2 \beta}\right) .
\end{aligned}
$$

Therefore

$$
\int_{s \leq|\xi| \leq 2 s}|\widehat{f}(\xi)|^{2} w_{k}(\xi) d \xi \leq C s^{-2 \alpha} \psi\left(s^{-2 \beta}\right) .
$$


Furthermore, we have

$\int_{|\xi| \geq s}|\widehat{f}(\xi)|^{2} w_{k}(\xi) d \xi$

$=\left(\int_{s \leq|\xi| \leq 2 s}+\int_{2 s \leq|\xi| \leq 4 s}+\int_{4 s \leq|\xi| \leq 8 s}+\ldots ..\right)|\widehat{f}(\xi)|^{2} w_{k}(\xi) d \xi$

$\leq C\left(s^{-2 \alpha} \psi\left(s^{-2 \beta}\right)+(2 s)^{-2 \alpha} \psi\left((2 s)^{-2 \beta}\right)+\left(2^{2} s\right)^{-2 \alpha} \psi\left(\left(2^{2} s\right)^{-2 \beta}\right)+\ldots \ldots\right)$

$\leq C\left(s^{-2 \alpha} \psi\left(s^{-2 \beta}\right)+2^{-2 \alpha} s^{-2 \alpha} \psi\left(s^{-2 \beta}\right)+\left(2^{-2 \alpha}\right)^{2} s^{-2 \alpha} \psi\left(s^{-2 \beta}\right)+\ldots.\right)$

$\leq C s^{-2 \alpha} \psi\left(s^{-2 \beta}\right)\left(1+2^{-2 \alpha}+\left(2^{-2 \alpha}\right)^{2}+\left(2^{-2 \alpha}\right)^{3}+\ldots ..\right)$

$\leq K_{\alpha} s^{-2 \alpha} \psi\left(s^{-2 \beta}\right)$,

where $K_{\alpha}=C\left(1-2^{-2 \alpha}\right)^{-1}$.

This proves that

$$
\int_{|\xi| \geq s}|\widehat{f}(\xi)|^{2} w_{k}(\xi) d \xi=O\left(s^{-2 \alpha} \psi\left(s^{-2 \beta}\right)\right) \text { as } s \longrightarrow+\infty
$$

2) $\Longrightarrow$ 1) Suppose now that

$$
\int_{|\xi| \geq s}|\widehat{f}(\xi)|^{2} w_{k}(\xi) d \xi=O\left(s^{-2 \alpha} \psi\left(s^{-2 \beta}\right)\right) \text { as } s \longrightarrow+\infty .
$$

We have to show that

$$
\int_{0}^{\infty} r^{2 \gamma+d-1}\left|1-j_{\gamma+\frac{d}{2}-1}(h r)\right|^{2 m} \phi(r) d r=O\left(h^{2 \alpha} \psi\left(h^{2 \beta}\right)\right),
$$

where we have set

$$
\phi(r)=\int_{\mathbf{S}^{d-1}}|\widehat{f}(r y)|^{2} w_{k}(y) d y
$$

We write

$$
I_{1}=\int_{0}^{1 / h} r^{2 \gamma+d-1}\left|1-j_{\gamma+\frac{d}{2}-1}(h r)\right|^{2 m} \phi(r) d r
$$

and

$$
I_{2}=\int_{1 / h}^{\infty} r^{2 \gamma+d-1}\left|1-j_{\gamma+\frac{d}{2}-1}(h r)\right|^{2 m} \phi(r) d r .
$$

Firstly, from (1) in lemma 1.3 we see that

$$
I_{2} \leq 4^{m} \int_{1 / h}^{\infty} r^{2 \gamma+d-1} \phi(r) d r=O\left(h^{2 \alpha} \psi\left(h^{2 \beta}\right)\right) \text { as } h \longrightarrow 0 .
$$

Set 


$$
g(r)=\int_{r}^{\infty} x^{2 \gamma+d-1} \phi(x) d x
$$

From (2) in lemma 1.3, an integration by parts yields

$$
\begin{aligned}
I_{1} & \leq-C_{1} h^{2 m} \int_{0}^{1 / h} r^{2 m} g^{\prime}(r) d r \\
& \leq-C_{1} g(1 / h)+2 m C_{1} h^{2 m} \int_{0}^{1 / h} r^{2 m-1} g(r) d r \\
& \leq 2 m C_{1} h^{2 m} \int_{0}^{1 / h} r^{2 m-1} r^{-2 \alpha} \psi\left(r^{-2 \beta}\right) d r \\
& \leq 2 m C_{1} h^{2 m} \int_{0}^{1 / h} r^{2 m-2 \alpha-1} \psi\left(r^{-2 \beta}\right) d r \\
& \leq C_{2} h^{2 m} h^{2 \alpha-2 m} \psi\left(h^{2 \beta}\right) \\
& \leq C_{2} h^{2 \alpha} \psi\left(h^{2 \beta}\right),
\end{aligned}
$$

where $C_{1}$ and $C_{2}$ are positive constants, and this ends the proof

\section{References}

[1] V. A. Abilov and F. V. Abilova, Approximation of Functions by Fourier-Bessel Sums, Izv. Vyssh. Uchebn. Zaved. Mat., No. 8, pp. 3-9 (2001).

[2] E. S. Belkina and S.S. Platonov, Equivalence of K-Functionals and Modulus of Smoothness Constructed by Generalized Dunkl Translations, Izv. Vyssh. Uchebn. Zaved. Mat, No. 8, pp. 3-15 (2008).

[3] C. F. Dunkl, Differential-difference operators associated to reflection group, Trans. Amer. Math. Soc. 311, pp. 167-183, (1989).

[4] C. F. Dunkl, Hankel transforms associated to finite reflection groups, Contemp. Math. 138, pp. 123-138, (1992).

[5] C. F. Dunkl, Integral kernels with reflection group invariance, Canad. J. Math. 43, pp. 1213-1227, (1991). 
[6] M. F. E. Jeu, The Dunkl transform, Invent. Math. 113, pp. 147-162, (1993).

[7] M. Maslouhi, An anlog of Titchmarsh's Theorem for the Dunkl transform, J. Integral. Trans. Spec. Funct, Vol. 21, Issue 10, pp. 771-778, (2010).

[8] M. Rösler, Posotivity of Dunkl's intertwining operator, Duke Math. J. 98, pp. 445-463, (1999). [arxiv.org/abs/q-alg/9710029].

[9] M. Rösler, M. Voit, Markov processes with Dunkl operators, Adv. in Appl. Math. 21, pp. 575-643, (1998).

[10] E. S. Titchmarsh, Introduction to the Theory of Fourier Integrals, Oxford University Press, Amen House, London, E. C. 4. (1948).

[11] K. Trimèche, Paley-Wiener theorems for the Dunkl transform and Dunkl translation operators, Integral Transforms Spec. Funct. 13, pp. 17-38, (2002).

\section{Radouan Daher}

Department of Mathematics, Faculty of Sciences Aïn Chock, University of Hassan II, Casablanca, Morocco e-mail: rjdaher024@gmail.com

and

\section{Mohamed El Hamma}

Department of Mathematics, Faculty of Sciences Aïn Chock, University of Hassan II, Casablanca, Morocco

e-mail: m_elhamma@yahoo.fr 